\title{
Algoritmos para Toma de Decisiones en Redes Inalámbricas Cognitivas: una Revisión
}

\author{
Diego A. Giral, Cesar A. Hernández* y Fredy H. Martinez \\ Universidad Distrital Francisco José de Caldas, Facultad Tecnológica, Bogotá-Colombia. \\ (e-mail: dagiralr@correo.udistrital.edu.co; cahernandezs@udistrital.edu.co; fhmartinezs@udistrital.edu.co) \\ * Autor a quien debe ser dirigida la correspondencia.
}

Recibido Abr. 5, 2019; Aceptado Jun. 12, 2019; Versión final Jul. 29, 2019, Publicado Dic. 2019

\begin{abstract}
Resumen
El objetivo de este artículo es presentar una revisión de técnicas basadas en inteligencia artificial para el proceso de toma de decisiones en redes de radio cognitiva. Las redes de radio cognitiva surgieron como una solución para resolver los problemas de asignación fija y de escasez del espectro, trabajan con un modelo de gestión que se denomina ciclo cognitivo, donde la toma de decisiones es clave, ya que permite seleccionar la oportunidad espectral más adecuada. A partir de literatura actualizada, se analizan estrategias que implementan máquinas de aprendizaje automático, algoritmos bioinspirados, teoría de juegos y algoritmos de consensos. Finalmente, el documento presenta los retos para el proceso de toma decisiones, donde se resalta la necesidad de seguir aprovechando los avances en inteligencia artificial para obtener mejores resultados. Sin embargo, las estrategias deben ser escalables para facilitar la carga computacional y poder resolver problemas de mayor complejidad.
\end{abstract}

\section{Algorithms for Decision Making in Wireless Cognitive Networks: Review}

\begin{abstract}
The objective of this article is to present a review of techniques based on artificial intelligence for the decisionmaking process in cognitive radio networks. The cognitive radio networks emerged as a solution to solve the problems of fixed allocation and scarcity of spectrum, work with a management model called the cognitive cycle. Decision making is key in this cycle since it allows to more appropriately selecting the spectral opportunity. From the current literature, strategies that implement machine learning, bioinspired algorithms, game theory and consensus algorithms are analyzed. Finally, this document presents the challenges for the decision-making process, which highlights the need to continue taking advantage of the advances in artificial intelligence to obtain better results. However, the strategies must be scalable to facilitate the computational load and being able to solve more complex problems.
\end{abstract}

Keywords: machine learning; artificial intelligence; bioinspired optimization; cognitive radio networks; decision making 


\section{INTRODUCCIÓN}

El número de dispositivos conectados a Internet ha aumentado en los últimos años y se proyecta que superará los 20 mil millones de dispositivos para 2020 (Gochoo et al., 2019). Según Cisco, el tráfico de datos móviles ha crecido 18 veces en los últimos 5 años, se estima que a nivel mundial aumente a un $46 \%$ entre 2017 y 2022, alcanzando 77.5 exabytes por mes para 2022 (CISCO, 2018). Adicionalmente, el uso del espectro libre, como ISM (industriales, científicas y médicas), ha aumentado en varias aplicaciones, como teléfonos inalámbricos, unidades remotas, Internet de las cosas y sistemas de audio y video (Pinto y Correia, 2018). Para resolver este problema, se ha propuesto el paradigma de la Radio Cognitiva (RC) como una estrategia para garantizar la calidad de la comunicación (Tripathi et al. 2019; Pinto y Correia, 2018). La RC fue presentada en 1999 como una nueva forma para abordar las redes de comunicación inalámbricas, Mitola y Maguire Jr., (1999) proponen una estructura eficiente, adaptativa y oportunista que permite realizar la asignación del espectro de forma dinámica.

A diferencia de las redes tradicionales, en la RC existen dos tipos de usuarios, el usuario que paga por utilizar una banda de frecuencia licenciada, denominado licenciado o primario (PU), y el usuario no licenciado 0 secundario (SU) que hace uso oportunista del espectro mientras esté disponible. La CR permite detectar, analizar y compartir el espectro, el objetivo es brindarle al SU espacios del espectro que no están siendo usados en ese momento por el PU, estos espacios se definen como oportunidades espectrales. La figura 1, muestra cómo un sistema basado en CR permite a los SU utilizar las oportunidades espectrales que están definidas por tiempo, frecuencia y potencia dinámica (Mitola y Maguire Jr., 1999; Ramzan et al., 2017).

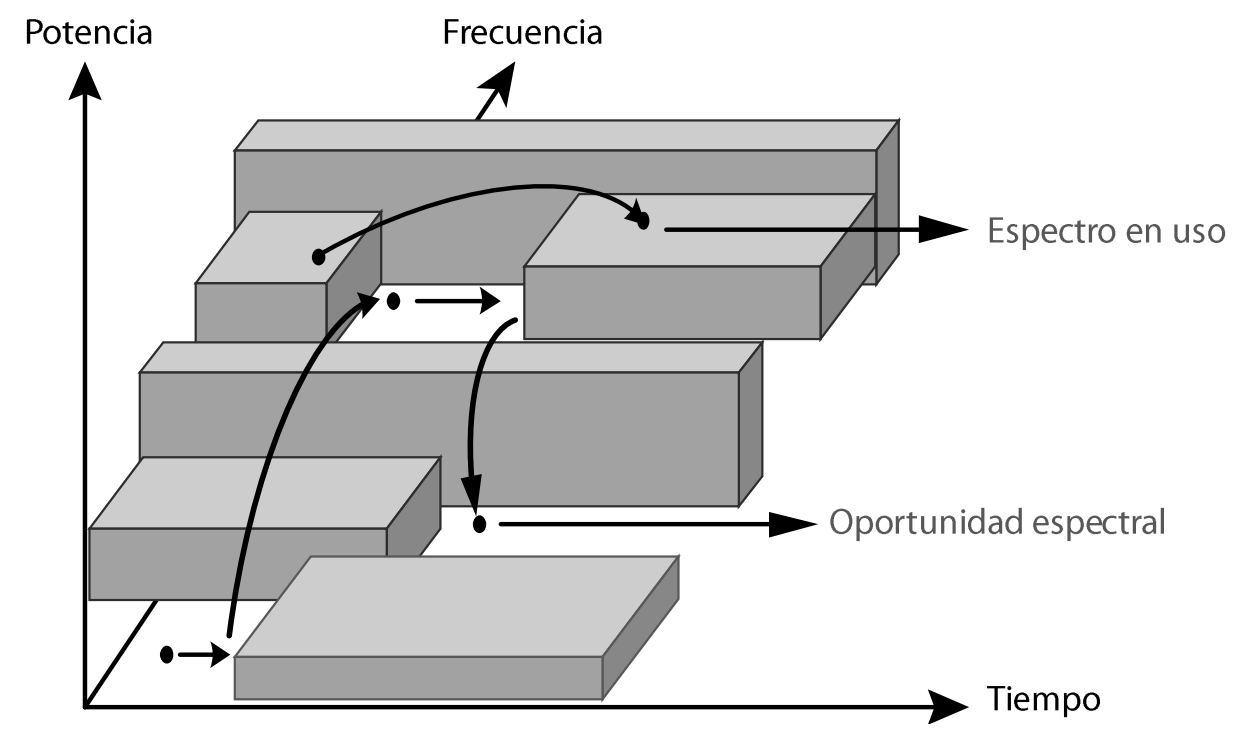

Fig. 1: Concepto de oportunidad espectral (Adaptado de López Sarmiento, 2017)

Para que se pueda implementar esta estructura, las Redes de Radio Cognitiva (CRN) trabajan con un modelo de gestión que se denomina ciclo cognitivo y se muestra en la figura 2. Mitola y Maguire Jr., (1999) delinearon el ciclo cognitivo como una estructura de radio que puede reconfigurarse mediante un proceso continuo de conciencia (tanto de sí mismo como del mundo exterior), percepción, razonamiento y decisión. El ciclo cognitivo se caracteriza por 4 elementos: detección del espectro, decisión del espectro, movilidad del espectro y la compartición del espectro (Masonta et al., 2013).

La decisión del espectro es una función clave, pero aún inexplorado en comparación con otros componentes del ciclo cognitivo. Después de realizar la detección del espectro, los SU deben decidir cuál es la mejor oportunidad espectral, de acuerdo con los requerimientos de calidad de servicio y a las acciones adoptadas por otros usuarios (Pedraza et al., 2019; Ramzan et al., 2017), las decisiones se deben tomar de forma inteligente en búsqueda de optimizar los resultados bajo las condiciones del entorno, esto se logra utilizando motor de decisión cognitivos o motores cognitivos. El motor cognitivo permite integrar algoritmos de inteligencia artificial de acuerdo a los parámetros de radio y al proceso de aprendizaje obtenido de la experiencia (Bernal y Hernández, 2019). Sin embargo, la integración para obtener un desempeño competente con una complejidad computacional mínima es una tarea difícil.

El objetivo del artículo es presentar una revisión de las técnicas basadas en inteligencia artificial para el proceso de toma de decisiones en CRN. El presente trabajo es una versión más completa, extendida y actualizada de la investigación realizada en (Hernández et al., 2016). El artículo, está estructurado de la 
siguiente forma, en la sección decisión espectral, se analizan las tres funciones principales para el proceso de toma de decisiones; en la sección algoritmos de toma de decisiones, se presenta la revisión de las técnicas de aprendizaje automático, algoritmos bioinspirados, teoría de juegos y algoritmos de consensos; en la sección comparación de los algoritmos, se presenta una tabla comparativa de las técnicas; en la sección retos en la decisión de espectro se analizan los retos actuales y las futuras orientaciones; finalmente, en la última sección, se presentan las conclusiones generales del trabajo.

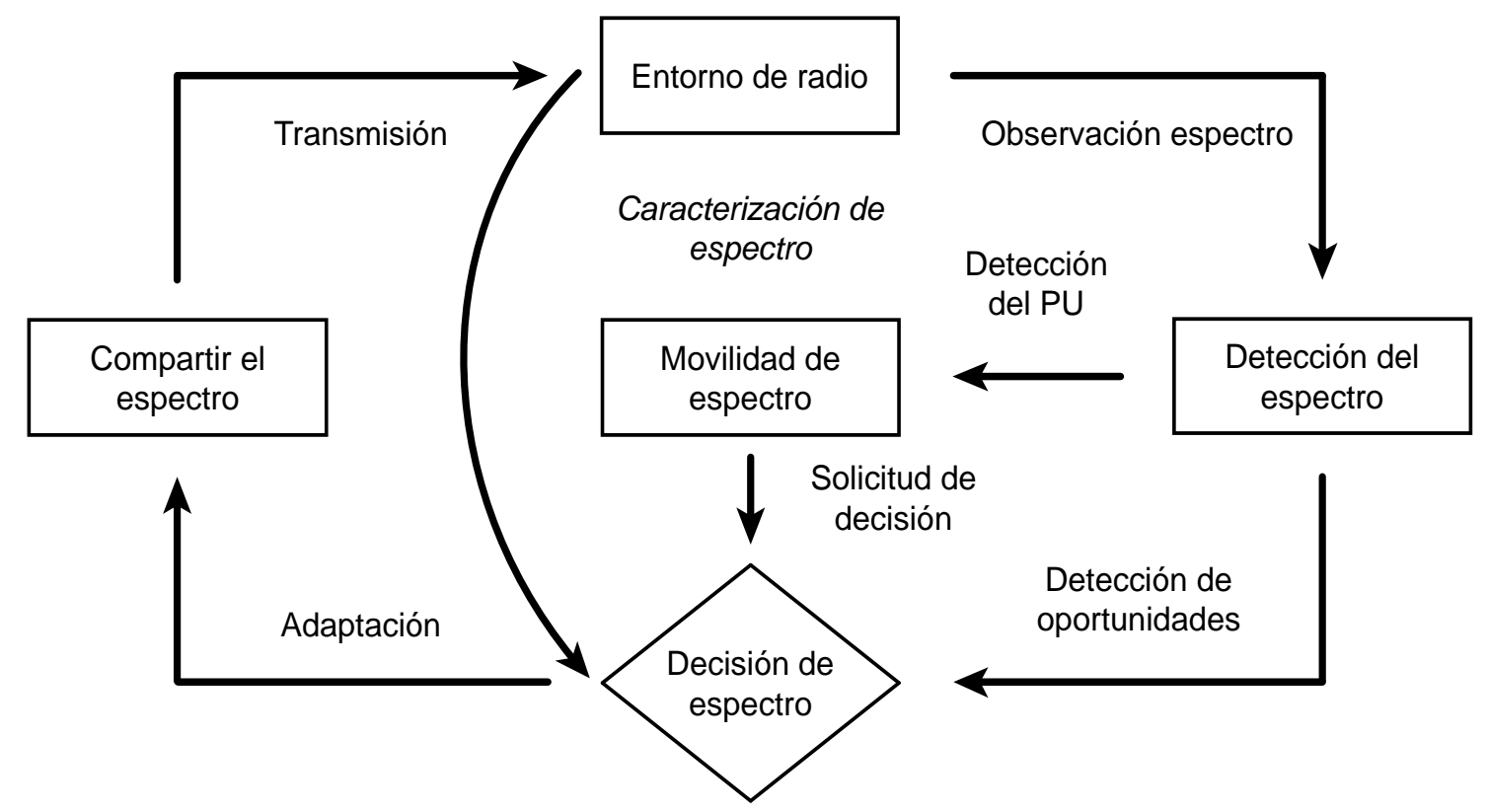

Fig. 2: Ciclo cognitivo (Adaptado de Ramzan et al., 2017)

\section{DECISIÓN ESPECTRAL}

La toma de decisiones en un proceso que busca seleccionar la mejor alternativa entre un conjunto finito de posibilidades, representa el corazón de los sistemas cognitivos, permite a los SU generar una secuencia de acciones que conducirán al logro de sus objetivos (Rizk et al., 2018; Tripathi et al., 2019). Para realizar estructuras de decisión es necesario implementar modelos con altos desafíos, los algoritmos deben ser escalables y eficientes, debido a los altos volúmenes de información que se requieren para el entrenamiento y validación, a la complejidad de las tareas y a los estándares de evaluación mínimos dé cada aplicación particular (Rizk et al., 2018).

La decisión espectral incluye tres funciones principales: (1) Caracterización del espectro: se identifican las bandas espectrales disponibles, normalmente denominada caracterización del PU; (2) Selección del espectro o decisión del espectro: selecciona la oportunidad espectral (canal de frecuencia) más adecuada, de acuerdo a los requerimientos del usuario; (3) Reconfiguración del CR: el CR debe ser capaz de reconfigurar los parámetros de su transceptor para soportar la comunicación dentro de la banda del espectro seleccionada. Como se muestra en la figura 3, una vez que los canales se identifican (utilizando sensores de espectro, bases de datos de geolocalización u otras técnicas) cada banda del espectro es caracterizada (actividad del PU) a partir de la base de observaciones locales y de la información estadística; culminada esta etapa los SU proceden a seleccionar la banda espectral más apropiada, a partir de la decisión tomada, el SU reconfigura sus parámetros de transmisión y continúa el envío de datos (López Sarmiento, 2017; Masonta et al., 2013).

\section{Caracterización del espectro}

En las CRN, para determinar correctamente la disponibilidad espectral más adecuada, se debe caracterizar cada una de las bandas disponibles, teniendo en cuenta factores como la intensidad de la señal, la interferencia y el número de PU. Son diversos los trabajos enfocados en la caracterización, López et al., (2017) lo analizan desde dos enfoques, basado en el modelo del PU y en el modelo del entorno de frecuencia (figura 4). El objetivo de la caracterización del PU o modelo de actividades, es predecir el comportamiento del PU para disminuir la probabilidad de que el PU y el SU se encuentren en el mismo canal durante el mismo instante de tiempo, fenómeno definido en las CRN como interferencia. La figura 4 presenta las técnicas utilizadas para la caracterización, en general están basadas en modelos de predicciones (López Sarmiento, 2017). 


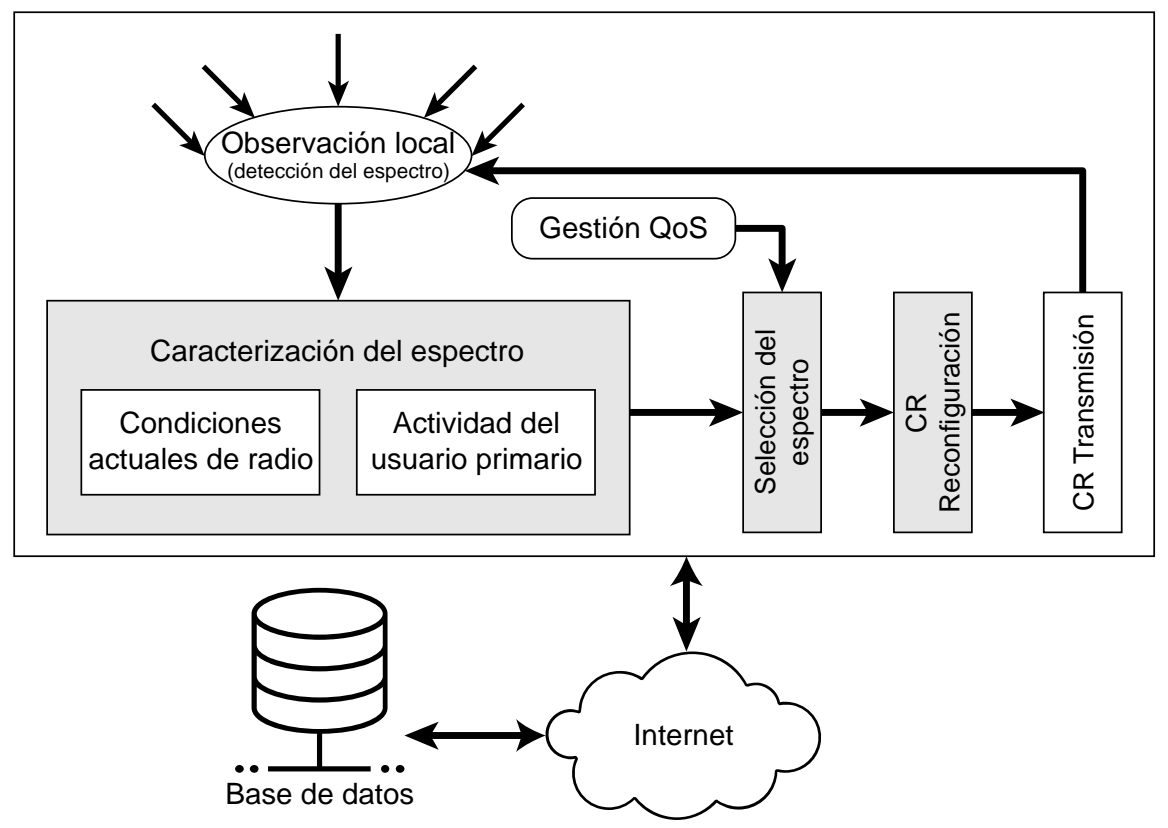

Fig. 3: Marco de decisión del espectro (Adaptado de López Sarmiento, 2017)

El éxito en la selección eficiente de canales dependerá de entorno, se espera que un CR caracterice continuamente a la radio en frecuencia, tiempo y espacio, los elementos o parámetros clave para la caracterización del entorno de radiofrecuencia se presentan en la figura 4 (Masonta et al., 2013). Como se muestra en la figura 4, las investigaciones están abiertas a nuevos desafíos, que permitirán mejorar la caracterización del espectro bajo parámetros más realistas.

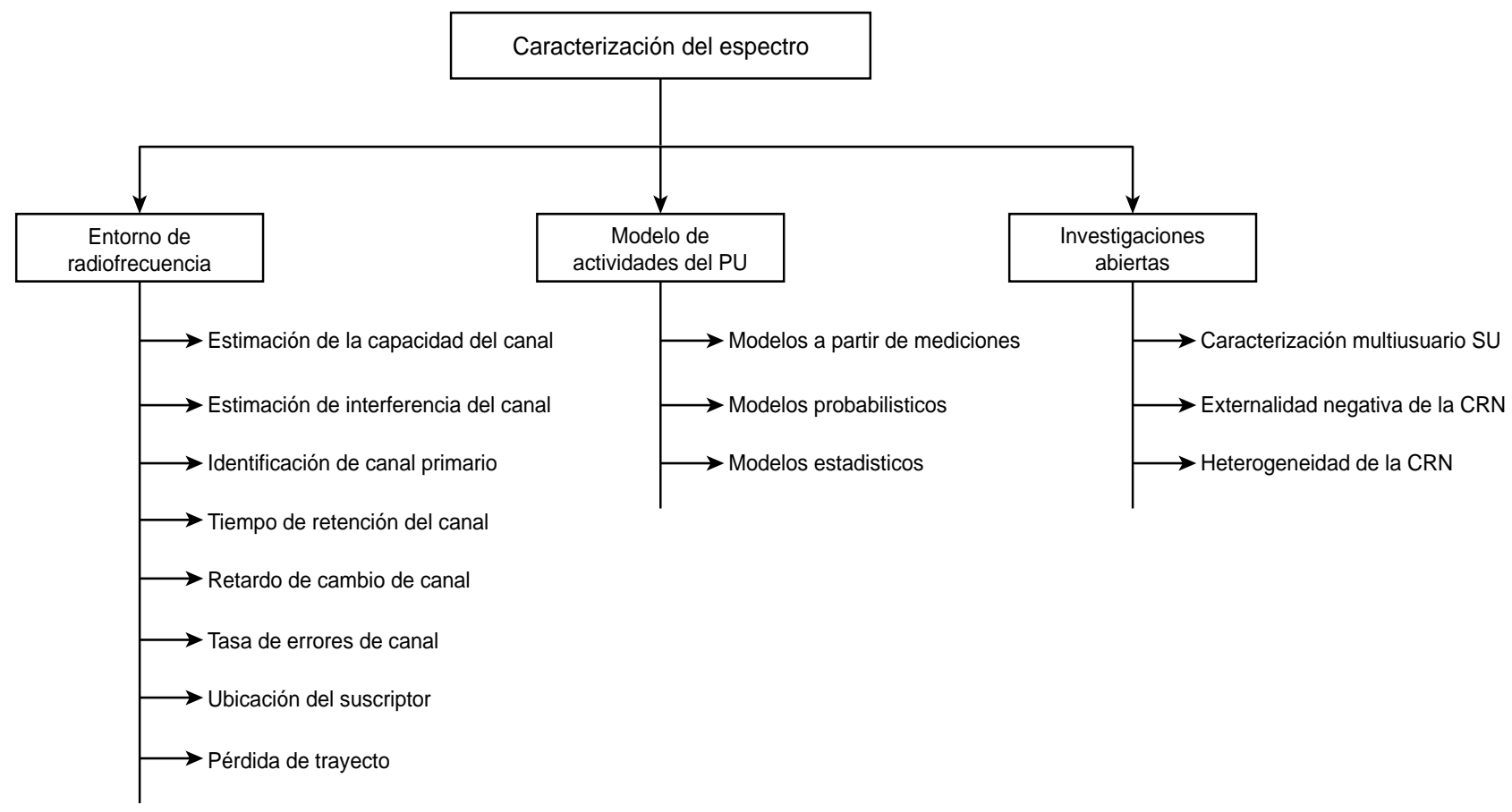

Fig. 4: Marco de caracterización del espectro (Adaptado de López et al., 2017; Masonta et al., 2013)

\section{Selección del espectro}

La selección del espectro, decisión del espectro o toma de decisiones es el resultado de la falta de conocimiento sobre el estado de los canales licenciados. De forma eficiente y sin causar ningún tipo de interferencia, establece mediante un conjunto de técnicas el proceso para seleccionar la oportunidad espectral más adecuada, de acuerdo con los requerimientos del SU y las condiciones del ambiente de radio. Un incorrecto proceso de toma de decisiones afecta los parámetros de la red como por ejemplo la tasa de cambios de canal o handoff espectral, sin embargo, a pesar de su relevancia no es una función tan explorada como la detección de espectro (Masonta et al., 2013; Pedraza et al., 2018). 
Los atributos de una CR, son la capacidad cognitiva, la reconfigurabilidad y la observación (Pedraza et al., 2019), estos tres atributos se incorporan en un elemento en común denominado motor cognitivo (CE). El CE explota el conocimiento sobre el entorno de radio circundante para optimizar el uso de los recursos de radio y adaptar los parámetros de transmisión relevantes (Alqerm y Shihada, 2017), se puede modelar como un agente inteligente que administra las tareas de cognición en una RC.

Las técnicas para la selección de espectro utilizan estrategias basadas en ciencias de la computación (Hernández et al., 2017; Abbas et al., 2015), entre las que se destaca la inteligencia artificial (IA), esta área ha permitido extender soluciones a campos como el aprendizaje automático, teoría de juegos, modelos probabilísticos, algoritmos de optimización, entre otros (Marquez et al., 2017). No hay un consenso entre los investigadores sobre lo que es IA, por lo tanto, no hay una definición exacta que permita dirimir que es o no inteligente. Se considera como IA el tratar de desarrollar instrucciones de una manera similar los métodos de pensamiento del cerebro humano (Bayrakdar y Çalhan, 2018).

La integración de la IA en redes inalámbricas es un elemento esencial, en el contexto de la gestión dinámica del espectro. Por los grandes volúmenes de información, la integración de dispositivos heterogéneos, la ultra densificación de los dispositivos, la interoperabilidad y la disipación de la batería, la IA es ampliamente utilizada en los procesos de toma de decisiones (Bayrakdar y Çalhan, 2018; Javaid et al., 2018; TuberquiaDavid et al., 2019; Bernal y Hernández, 2019).

\section{Reconfiguración del espectro}

En las redes inalámbricas tradicionales, los terminales de radio son estáticamente configurados para operar sobre canales de frecuencia predefinidos. La reconfigurabilidad es la capacidad de cambiar de forma dinámica, los diferentes parámetros de operación relacionados con la modulación, potencia de transmisión, frecuencia de operación, banda ancha del canal y tecnología de la comunicación, esta función se realiza después de haber caracterizado y seleccionado el espectro. Esta capacidad le permite a las CRN una mayor adaptación al entorno radio dinámico. La tabla 1 proporciona la descripción de los parámetros de reconfiguración con las técnicas existentes (Pedraza et al., 2019; Masonta et al., 2013).

Tabla 1: Soluciones de reconfiguración de parámetros (Masonta et al., 2013; Pedraza et al., 2019)

\begin{tabular}{|l|l|l|}
\hline \multicolumn{1}{|c|}{ Parámetros } & \multicolumn{1}{|c|}{ Técnicas existentes } & \multicolumn{1}{c|}{ Comentarios } \\
\hline $\begin{array}{l}\text { Modulación y } \\
\text { codificación }\end{array}$ & Esquema de transmisión adaptable & $\begin{array}{l}\text { Para CRN centralizadas con } \\
\text { temperatura de interferencia }\end{array}$ \\
\hline $\begin{array}{l}\text { Potencia de } \\
\text { transmisión }\end{array}$ & $\begin{array}{l}\text { Sistema conjunto de control de } \\
\text { admisión y asignación de energía }\end{array}$ & $\begin{array}{l}\text { Para las estimaciones CRAHN } \\
\text { y SU, los límites de interferencia } \\
\text { en el receptor PU }\end{array}$ \\
\hline $\begin{array}{l}\text { Frecuencia de } \\
\text { operación }\end{array}$ & $\begin{array}{l}\text { Modelo predictivo para seleccionar } \\
\text { dinámicamente la frecuencia de } \\
\text { funcionamiento correcta }\end{array}$ & $\begin{array}{l}\text { Requiere una actualización } \\
\text { continua para asegurar la } \\
\text { predicción en tiempo real }\end{array}$ \\
\hline $\begin{array}{l}\text { Banda ancha del } \\
\text { canal }\end{array}$ & $\begin{array}{l}\text { Esquema de asignación de ancho de } \\
\text { banda adaptable basado en colas }\end{array}$ & $\begin{array}{l}\text { Ayuda al distribuidor del canal a } \\
\text { decidir la cantidad de ancho de } \\
\text { banda para el SU en redes } \\
\text { centralizadas. }\end{array}$ \\
\hline $\begin{array}{l}\text { Tecnología de la } \\
\text { comunicación }\end{array}$ & $\begin{array}{l}\text { Sistema de radio reconfiguración } \\
\text { basado en red móvil }\end{array}$ & $\begin{array}{l}\text { Permite que la RC opere bajo } \\
\text { diferentes tecnologías de } \\
\text { acceso de radio e incluye auto- } \\
\text { organización dinámica. }\end{array}$ \\
\hline
\end{tabular}

\section{ALGORITMOS DE DECISIÓN DE ESPECTRO}

En la literatura se encuentran diferentes técnicas de inteligencia IA para la toma de decisiones (Hernández et al., 2016). La figura 5 muestra una comparación entre los diferentes modelos para toma de decisiones basado en tres criterios: 1) heterogeneidad; 2) escalabilidad; y 3) ancho de banda de comunicación. En las secciones siguientes se realiza una descripción de las técnicas basadas en máquinas de aprendizaje automático, algoritmos bioinspirados, teoría de juegos y algoritmos de consensos. 


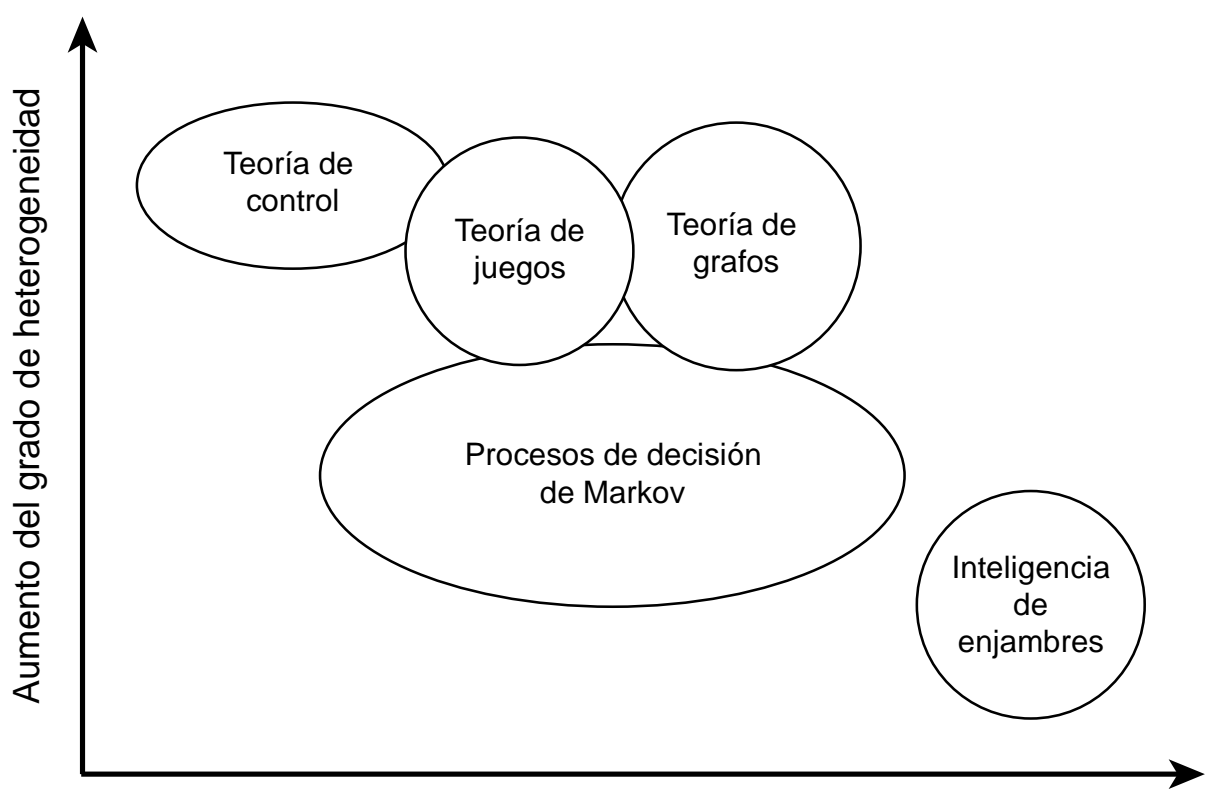

Aumento del grado de escalabilidad

Fig. 5: Comparación de los marcos de modelos de toma de decisión (Adaptado de Rizk et al., 2018)

\section{Aprendizaje automático}

El aprendizaje automático (machine learning) nació del reconocimiento de patrones, se basa en la premisa de que las máquinas deben contar con inteligencia artificial, que les permita aprender de cálculos anteriores y adaptarse a su entorno a través de la experiencia.

Los algoritmos de aprendizaje en las CRN están integrados en todos los procesos de adquisición de conocimiento para definir la estructura de toma de decisiones (Jayaweera, 2014). Para que los algoritmos puedan aprender existen dos tipos de estrategias: aprendizaje supervisado y no supervisado. Para aprendizaje supervisado en CR se encuentran modelos basados en redes neuronales y máquinas de soporte vectorial (Jayaweera, 2014), en estrategias no supervisadas, el aprendizaje por refuerzo es una técnica que permite a los usuarios modificar su comportamiento al interactuar con su entorno (Sutton y Barto, 2018).

Un ciclo cognitivo basado en el aprendizaje por refuerzo para $\mathrm{CRN}$, con agentes múltiples que interactúan entre sí, en un entorno común sin estación base se presenta en la figura 6 . En cada ciclo de aprendizaje, los usuarios reciben una observación parcial del estado actual con una recompensa acumulada. Al utilizar la observación del estado y el valor de recompensa, el usuario actualiza su política durante la etapa de aprendizaje. Finalmente, durante el proceso de toma de decisiones, cada usuario selecciona una acción óptima de acuerdo a las acciones óptimas tomadas por el conjunto de usuarios (Yonghua Wang et al., 2019; Bkassiny et al., 2013).

Es imposible enumerar todos los aprendizajes que pueden surgir dentro de una $\mathrm{CRN}$, por lo tanto, el trabajo permanente en el proceso de toma de decisiones es identificar el impacto de las diferentes estrategias. Generalmente, las técnicas de aprendizaje automático en CRN se utilizan para mejorar las métricas de calidad de servicio. Una de sus ventajas es la capacidad de modelar el comportamiento de la red en intervalos futuros, permitiendo la aplicación de soluciones proactivas. Sin embargo, los problemas a solucionar se pueden complicar exponencialmente, por ejemplo, si se analizan CRN heterogéneas, para este caso, además del reto de lograr adaptarse al entorno de radio frecuencia, el modelo propuesto debe coordinar sus acciones con respecto a las otras radios en la red (Bkassiny et al., 2013).

Bkassiny et al., (2013) realiza la descripción de algunas técnicas basadas en algoritmos de aprendizaje automático según el problema de CR a solucionar. La organización jerárquica de los algoritmos de aprendizaje y su dependencia se muestra en la figura 7 , se identifican dos categorías principales: toma de decisiones y clasificación de las características, las categorías se analizan de forma general para cubrir un amplio espectro de problemas. Por ejemplo, los problemas de clasificación surgen en la detección del espectro, mientras que los problemas de toma de decisiones surgen al determinar la política de detección del espectro, el control de potencia o la modulación adaptativa. 


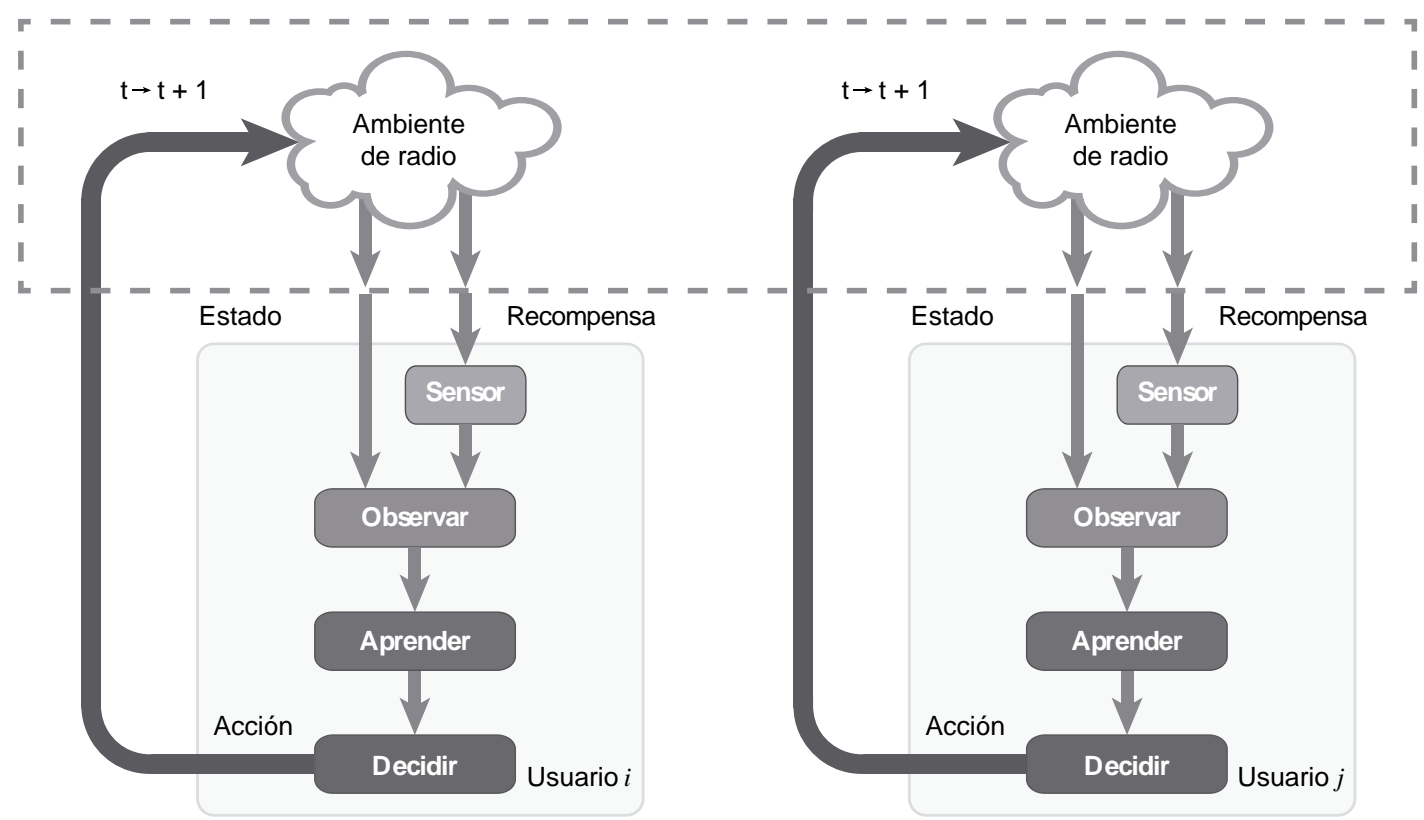

Fig. 6: Ciclo cognitivo basado en el aprendizaje por refuerzo para usuarios múltiples sin base central (Adaptado de Abbas et al., 2015)

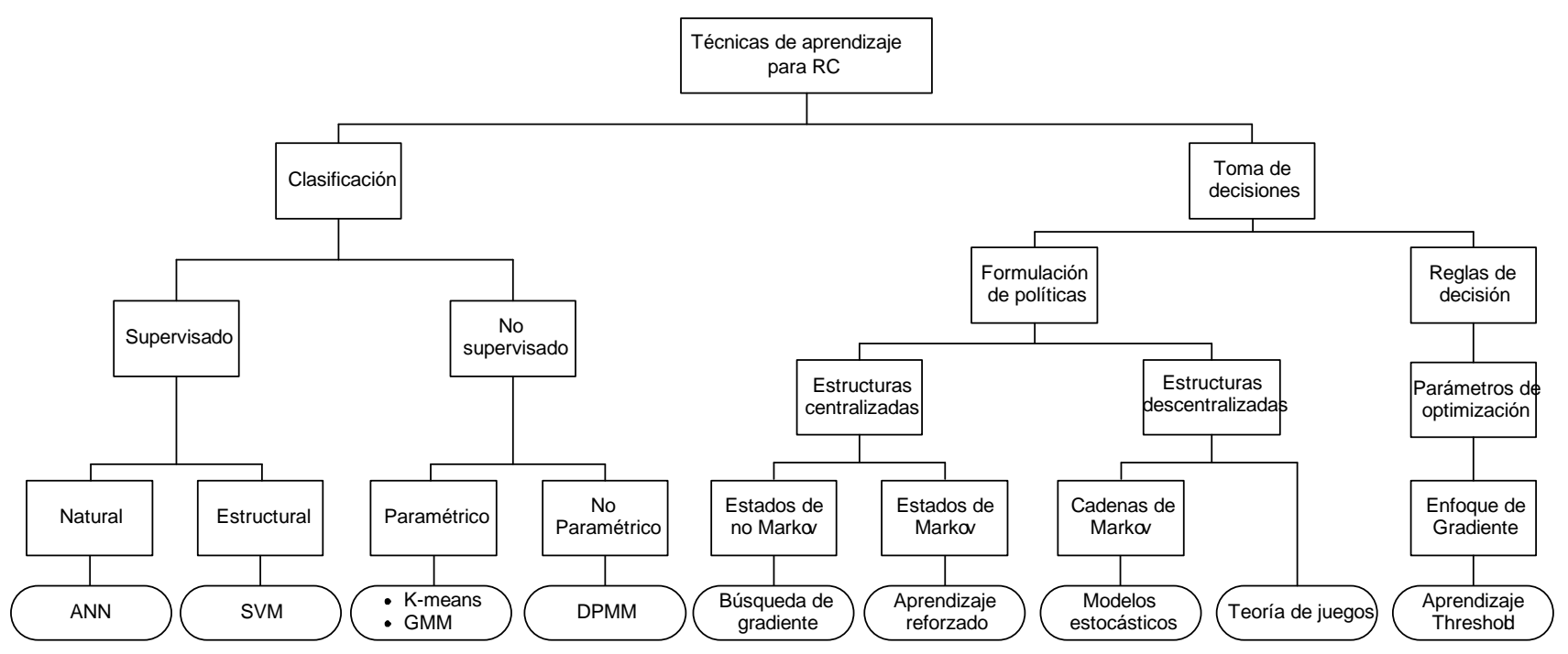

Fig. 7: Problemas típicos en RC y sus correspondientes algoritmos de aprendizaje (Adaptado de Bkassiny et al., 2013)

La figura 7 presenta algunos de los modelos de aprendizaje automático, sin embargo, en la literatura se encuentran disponible un número amplio de técnicas, algunas totalmente nuevas que surgen de propuestas de los autores para aplicaciones específicas (Tripathi et al., 2019; Shi et al., 2018) y otras basadas en modelos híbridos, estas propuestas utilizan el principio de "free lunch" (Wu et al., 2019), caracterizan las ventajas y desventajas de 2 o más estrategias para luego combinarlas de tal forma que el algoritmo global sea mejor que los individuales (Shi et al., 2018; Hernández et al., 2017).

\section{Algoritmos bioinspirados}

La investigación de operaciones modela y resuelve problemas complejos de toma de decisiones a través de algoritmos de optimización. Estos algoritmos han sido ampliamente estudiados por los investigadores durante mucho tiempo (Li et al., 2016). La computación bioinspirada es una rama de la optimización que toma como fuente de inspiración los fenómenos naturales (Bozorg-Haddad, 2018). Esta estrategia conocida como optimización metaheuristica, ha enriquecido las técnicas de optimización, debido a que presenta rendimientos eficientes, y soluciones viables para los complejos problemas presentes en la investigación de operaciones (Li et al., 2016), además, son ampliamente utilizadas por su simplicidad y flexibilidad (Bernal y Hernández, 2019). 
Por ejemplo, Alqerm y Shihada, (2014a) y Alqerm y Shihada, (2014b) propone un esquema de optimización de objetivos múltiples adaptativo para mejorar la eficiencia del sistema con una versión mejorada del algoritmo genético (GA). A pesar del poder de GA en la optimización de objetivos múltiples, su complejidad y la convergencia relativamente lenta limita su rendimiento. Hasegawa et al., (2014) propone algoritmos de optimización para la toma de decisiones en redes inalámbricas cognitivas heterogéneas, utilizando como estrategia una red neuronal Hopfield-Tank, la propuesta es validada mediante un conjunto de simulaciones para finalmente ser implementada en un sistema cognitivo experimental. Otras técnicas metaheurísticas inspiradas en la naturaleza son las basadas en los modelos de enjambres, permiten analizar el comportamiento colectivo descentralizado y autoorganizado. Los sistemas auto-organizados con inteligencia de enjambres como posible solución han atraído la atención de la academia y de la industria (Peška et al., 2019; Bozorg-Haddad, 2018). Los sistemas basados en la inteligencia de enjambre son muy flexibles y robustos con respecto a las limitaciones y perturbaciones ambientales (Murmu y Singh, 2019), son una tendencia emergente con varias áreas de aplicación (Peška et al., 2019), por ejemplo, la optimización de enjambre de partículas (PSO) y la optimización de colonias de hormigas (ACO) se considera una excelente herramienta para la implementaciones de motores cognitivos (Alqerm y Shihada, 2017).

Desdé el punto de vista de las redes de telecomunicación, los sistemas basados en inteligencia de enjambre son muy flexibles y robustos (Murmu y Singh, 2019), presentan ventajas importantes como: (1) Escalabilidad: el número de personas puede adaptarse al tamaño de la red; (2) Tolerancia a fallos: dado que el comportamiento de un enjambre no está controlado por una entidad centralizada, la pérdida de unos pocos individuos no causa un fracaso catastrófico; (3) Adaptación: el enjambre puede reaccionar a los cambios ambientales debido al hecho de que cada individuo tiene la capacidad de adaptarse, conduciendo a un alto valor de flexibilidad; (4) Velocidad: los cambios en la red pueden propagarse muy rápidamente entre los usuarios de la red. (5) Modularidad: los individuos actúan independientemente de otras capas de la red; (6) Autonomía: se requiere poco o ningún control humano, conduciendo a una velocidad de convergencia mucho más rápida; (7) Paralelismo: las operaciones de las personas se realizan de forma paralela.

Las técnicas de optimización Genetic Algorithm (GA) y Particle Swarm optimization (PSO) son algoritmos de búsqueda estocástica que emulan la evolución natural y el comportamiento de la inteligencia de enjambre respectivamente, se han aplicado con éxito en muchos campos de investigación asociadas a procesos de toma de decisiones. Kaur et al., (2018) proponen un módulo de toma de decisiones para RC llamado Cognitive Decision Engine (CDE), este módulo es diseñado para adaptarse y reconfigurarse a los parámetros de transmisión con el objetivo de satisfacer los requisitos de calidad de servicio. La investigación presenta el análisis comparativo del rendimiento de la optimización multiobjetivo para un motor cognitivo utilizando Algoritmo Genético codificado real (RCGA) y un motor cognitivo utilizando enjambre de partículas (PSO).

La transferencia de espectro (SHO) es un reto interesante para las CRN. Modi y Murmu, (2017) formula un esquema de transferencia múltiple del espectro de SU utilizando optimización de colonias de hormigas (ACO). El esquema formula una función de costo que ayuda a tomar la decisión de traspaso y realiza una asignación justa de canales para mantener los requisitos de calidad de servicio en CRN. Li et al., (2016) propone un algoritmo de búsqueda autónomo (ASA), basado en la evolución de la población, reproducción, selección y mutación. El modelo propuesto es comparado con un algoritmo de optimización por enjambre de partículas (PSO) y un algoritmo genérico (AG). Los resultados experimentales muestran que ASA satisface la demanda de comunicación y tiene un buen rendimiento con respecto a las otras estrategias analizadas. Puede optimizar adaptablemente los parámetros de transmisión de acuerdo con las condiciones del canal y el tipo de cambio en el servicio al cliente, lo que le permite obtener un mejor esquema de decisión de parámetros.

Durante los últimos años, un considerable número de nuevos algoritmos bioinspirados se han propuesto en la literatura, sin embargo, aunque la mayoría de estos algoritmos son muy prometedores, la mayoría están en su infancia (Bozorg-Haddad, 2018). A partir del teorema de "No free lunch" se sabe que no se puede encontrar ningún algoritmo metaheurístico que sea el mejor para cualquier problema, si un algoritmo supera a otros en alguna función, habrá otra función de optimización en las que otros algoritmos serán mejores que los anteriores algoritmos (Wu et al., 2019).

Bozorg-Haddad, (2018) en su libro "Advanced optimization by nature-inspired algorithms" presenta las nuevas propuestas de algoritmos bioinspirados desarrolladas para un periodo entre el 2007 y 2016, los modelos descritos son útiles en estudios multidisciplinares, incluyendo el proceso de toma de decisiones en CRN, cada capítulo del libro describe un algoritmo: optimización de enjambres de gatos (CSO), algoritmo de campeonato de la liga (LCA), optimización de la sociedad de anarquías (ASO), algoritmo de optimización de cuco (COA), optimización basada en el aprendizaje del profesor (TLBO), algoritmo de polinización de flores (FPA), algoritmo de la manada de krill (KHA), optimización del lobo gris (GWO), optimización del olor a tiburón (SSO), optimización del león hormiga (ALO), evolución del gradiente (GE), optimización de la polilla y la llama (MFO), algoritmo de búsqueda de la médula espinal (CSA) y el algoritmo de la mosca de los dragones (DA). 


\section{Teoría de juegos}

La teoría de juegos, fue presentada por John von Neumanny y Oskar Morgenstern en "The Theory of Games Behavior" publicado en 1953 (Morgenstern y Von Neumann, 1953), es una estrategia de análisis, que estudia las características generales de situaciones competitivas que surgen de interacciones estratégicas entre jugadores racionales, y proporciona herramientas de modelamiento para comprender y analizar los problemas de toma de decisión (Ren y Wong, 2019; Darwish et al., 2018; Rizk et al., 2018). La teoría de juegos es un modelo matemático que analiza interacciones mutuas en sistemas de decisión multiusuario. Consta de un número fijo de jugadores, comandos de acción y una función de utilidad que asigna las acciones del jugador a un valor real. Además, las decisiones de los actores son distribuidas y autónomas, lo que conduce a una optimización auto-organizada.

Un juego puede ser representado matemáticamente mediante la ecuación (1).

$$
G=\left\{N, A,\left\{u_{i}\right\}\right\}
$$

En esta ecuación,

$N=\{1,2, \ldots, N\}$ es el conjunto finito de jugadores

$A=A_{1} \times A_{2} \times \ldots \times A_{1}$ es el conjunto de acciones, $A_{i}$ conjunto de acciones del jugador $i$.

$u_{i}: A \rightarrow R$ es la función de pago/utilidad del jugador $i$.

Los tres componentes principales de un modelo basado en teoría de juegos son: los jugadores (los que toman las decisiones), el conjunto de acciones (las alternativas disponibles) y la función objetivo. La teoría de juegos no estudia la probabilidad de los resultados de ocurrencia de un juego, su objetivo principal, es analizar el comportamiento estratégico entre jugadores, por eso, es una estrategia útil en el proceso de toma decisiones y atractiva para diversos campos de las ciencias de la información (Tabassam y Suleman, 2012).

Las estrategias basadas en juegos proporcionan un marco ideal para modelar y analizar los sistemas de comunicación (Tabassam y Suleman, 2012). En CRN, los usuarios de la red deben tomar decisiones inteligentes basados en la dinámica del espectro y en las acciones adoptadas por otros usuarios. Por lo tanto, es natural estudiar el comportamiento e interacciones de los usuarios de CRN desde un punto de vista de teoría de juegos (Song et al., 2019).

Los jugadores del juego (nodos de red) tendrían el conocimiento completo, parcial o sin conocimiento mutuo en un espacio de acción (entorno de red) trabajando en una teoría de juego competitiva o cooperativa. En cualquier juego, la utilidad (resultado) representa la motivación de los jugadores, mientras que la función de utilidad mapea el tipo y la elección de acciones (estrategias) por parte de todos los jugadores a un número real (Shattal et al. 2018). La estrategia de cada jugador (nodo de radio) debe maximizar la utilidad esperada para obtener la mejor respuesta de cada jugador para un equilibrio bien definido. En la tabla 2 se describe los elementos de un juego para redes inalámbricas basado en RC (Garg et al., 2015; Tabassam y Suleman, 2012). La teoría de juegos se divide en dos categorías, los juegos cooperativos y no cooperativos (Garg et al., 2015).

Tabla 2: Elementos de un juego en CR (Garg et al., 2015)

\begin{tabular}{|l|l|l|}
\hline Componente del juego & \multicolumn{1}{|c|}{ Descripción } & $\begin{array}{c}\text { Elemento modelado de } \\
\text { CRN }\end{array}$ \\
\hline Jugadores & $\begin{array}{l}\text { Los jugadores deben tratar de maximizar } \\
\text { su función de utilidad considerando la } \\
\text { actividad de los usuarios primarios. }\end{array}$ & Nodos en red inalámbrica \\
\hline $\begin{array}{l}\text { Estrategia/Conjunto de } \\
\text { acciones }\end{array}$ & $\begin{array}{l}\text { Acciones que están relacionadas con la } \\
\text { funcionalidad que se está observando. }\end{array}$ & $\begin{array}{l}\text { Esquema de modulación, } \\
\text { velocidad de codificación, } \\
\text { asignación de canales, } \\
\text { nivel de potencia de } \\
\text { transmisión, selección de } \\
\text { rutas, etc. }\end{array}$ \\
\hline $\begin{array}{l}\text { Función de utilidad/ } \\
\text { Conjunto de } \\
\text { preferencias }\end{array}$ & $\begin{array}{l}\text { El objetivo del jugador que mide el } \\
\text { resultado para un jugador de RC. }\end{array}$ & $\begin{array}{l}\text { Métricas de rendimiento, } \\
\text { por ejemplo, rendimiento, } \\
\text { retardo, SINR, QoS, etc. }\end{array}$ \\
\hline
\end{tabular}




\section{Juegos cooperativos}

Analiza la formación de grupos de jugadores que cooperan entre sí para maximizar la utilidad de la CR. En cada coalición o cooperación, los jugadores coordinan sus acciones y agrupan sus ganancias para fortalecer la posición de los miembros del grupo en el juego. Hassan et al., (2016) proponen dos estrategias de toma de decisiones autónomas para ayudar a cada usuario de la CR a decidir si participar o no en la transmisión cooperativa, las estrategias están basadas en pruebas de arrepentimiento (RTS) y en el aprendizaje (LBS). La recompensa adquirida y su costo pagado son los incentivos para que los usuarios de CR participen en la transmisión cooperativa, las estrategias de toma de decisiones de participación propuestas se extienden para casos con múltiples usuarios que requieren cooperación simultáneamente.

\section{Juegos no cooperativos}

En los cuales los jugadores son usuarios egoístas y toman acciones de forma independiente con el objetivo de maximizar sus propias funciones de utilidad. La teoría de juegos no cooperativa nos permite derivar enfoques distribuidos eficientes para compartir el espectro dinámico usando solo información local. Los modelos de teoría de juegos no cooperativos se han desarrollado para comprender mejor el control de la congestión, el enrutamiento, el control de potencia, el control de topología, la gestión de confianza y muchos otros problemas en los sistemas de comunicación inalámbricos (Garg et al., 2015). Safwat y Elbadawy, (2018) analizan el problema de toma de decisiones para acceder a las CRN utilizando modelos de teoría de colas y de juegos. La interacción entre los usuarios cognitivos se trata como un juego no cooperativo. Se deriva una expresión de forma cerrada para el número promedio de usuarios cognitivos, así como el tiempo de espera esperado asociado en los CRN superpuestos y subyacentes. A través del análisis proporcionado se demuestra que se puede obtener el equilibrio de Nash o equilibrio de Cournot y Nash.

Debido a la movilidad de los nodos y la dinámica de la variación del canal, la precisión de las decisiones de los usuarios es limitada y sigue siendo un desafío para utilizar plenamente los escasos recursos de espectro. La mayoría de los enfoques suponen que la utilidad de un SU es independiente de las decisiones tomadas por otros SU, sin embargo, tal suposición no es cierta, especialmente cuando se consideran escenarios en los que los SU comparten o compiten por ciertos recursos (Masonta et al., 2013).

Las comunicaciones inalámbricas entre múltiples usuarios, es un reto para los sistemas de próxima generación (Jiang et al., 2014a). Al tomar la decisión de acceso al canal, cada SU no solo debería considerar la calidad del canal, sino que también debe tener en cuenta las decisiones de acceso del canal de otros SU, cuanto más acceden los SU al mismo canal, menor es el rendimiento que pueden alcanzar debido a la interferencia entre ellos, este fenómeno se conoce como externalidad negativa de la red (Jiang et al., 2014a). La teoría de juegos proporciona un medio elegante para modelar la interacción estratégica entre sistemas multiagente que pueden o no ser de naturaleza cooperativa.

Zhang et al., (2015) formulan el proceso de aprendizaje y de toma de decisiones de los SU como un juego de "Chinese restaurant", donde se analiza la externalidad negativa de la red, se aborda el problema de la decisión de acceso secuencial, un modelo más realista, pero con mayores dificultades para las CRN. En el juego propuesto, los SU detectan simultáneamente los canales para estimar el estado y luego de forma secuencial toman la decisión del canal a acceder. Una vez que un SU selecciona un canal, la decisión es revelada a través de un canal de control de comunicación a los demás SU

\section{Algoritmos de consensos}

Según la teoría clásica de la decisión (Luce y Raiffa, 1958) un problema de decisión se puede clasificar en: toma de decisión individual y toma de decisión en grupo (TDG). La TDG es un problema en el que los expertos intentan lograr una solución común, teniendo en cuenta las opiniones o preferencias de todos los involucrados, sin embargo, algunas de las decisiones tomadas no son aceptadas como buenas por parte de algunos de los expertos. Para evitar estos desacuerdos, se requiere de un proceso de consenso, en el que los expertos discuten y modifican sus preferencias para alcanzar un nivel de acuerdo antes de tomar la decisión. El consenso se define como un acuerdo donde los expertos alinean sus posiciones para tomar una decisión. El concepto de consenso se ha definido y estudiado de diferentes maneras en la literatura: el consenso se define como una opinión grupal, y se refiere a los enfoques, modelos, herramientas y procedimientos donde los expertos alinean sus posiciones para tomar una decisión grupal (Zhang et al. 2019).

Nguyen y Ahn, (2018) presentan una hibridación entre algoritmos de consenso e inclusiones diferenciales para resolver de manera efectiva los juegos potenciales exactos con un espacio de estrategia continua en una red de múltiples agentes. Wang y Djurić, (2013) abordan el problema de alcanzar un consenso bayesiano en un sistema cognitivo modelado por un conjunto de agentes distribuidos espacialmente. Se propone un 
método que permita a los agentes alcanzar un consenso local óptimo mediante el intercambio de decisiones. Con la estrategia propuesta, todos los agentes del sistema pueden lograr un consenso en la decisión, que además satisface la decisión óptima global.

\section{COMPARACIÓN DE LOS ALGORITMOS}

Existen un alto número de trabajos que analizan o recopilan estrategias para el proceso de toma de decisiones, sin embargo, como se describió en secciones previas y a partir del teorema de "No free lunch", no hay una técnica que sea la mejor, cada estructura presenta un número considerable de ventajas y desventajas, lo que permite caracterizarlas para problemas específicos. En la revisión presentada, actualmente la hibridación está generando soluciones con buenos resultados. Los retos están en la carga computacional, los algoritmos para toma de decisiones no solo deben entregar buenos resultados y resolver tareas complejas, deben diseñarse para que sean eficientes. A partir de la información analizada, la tabla 3 presenta la descripción de las técnicas de acuerdo con algunos algoritmos, se utilizan como características, la complejidad computacional, la precisión, la estrategia, y el aprendizaje. Adicionalmente, en la última columna de la tabla 3 se presentan algunas aplicaciones según el tipo de algoritmo, esta descripción es de carácter general, el proceso de toma de decisiones es una función que debe trabajar de forma coordinada con los demás componentes del ciclo cognitivo. Las CRN son la estrategia para resolver ecosistemas basados en modelos inteligentes como: negocios, educación, agricultura, monitoreo, seguridad, hogar, salud, electricidad, tráfico vehicular, entre otros.

Tabla 3: Descripción de las técnicas para toma de decisiones basadas en inteligencia artificial (CC=Complejidad computacional).

\begin{tabular}{|c|c|c|c|c|c|c|c|}
\hline \multirow{2}{*}{ Técnica } & \multirow{2}{*}{ Algoritmo } & \multicolumn{4}{|c|}{ Característica } & \multirow{2}{*}{ Referencia } & \multirow{2}{*}{ Aplicación } \\
\hline & & $C C$ & Precisión & Estrategia & Aprendizaje & & \\
\hline $\begin{array}{l}\text { Aprendizaje } \\
\text { automático }\end{array}$ & $\begin{array}{l}{ }^{*} \text { Redes } \\
\text { neuronales } \\
\text { artificiales } \\
\text { *Máquinas de } \\
\text { soporte } \\
\text { vectorial } \\
\text { *Aprendizaje } \\
\text { por refuerzo } \\
\text { *Aprendizaje } \\
\text { multiagente }\end{array}$ & Alta & Alta & $\begin{array}{c}\text { Colaborativa } \\
\text { No } \\
\text { colaborativa }\end{array}$ & $\begin{array}{l}\text { Supervisado } \\
\text { No } \\
\text { supervisado }\end{array}$ & $\begin{array}{l}\text { (Jayaweera, } \\
\text { 2014; Abbas } \\
\text { et al., 2015; } \\
\text { López et al., } \\
\text { 2017; Rizk et } \\
\text { al., 2018; } \\
\text { Sutton y Barto, } \\
\text { 2018;Yonghua } \\
\text { Wang et al., } \\
\text { 2019) }\end{array}$ & $\begin{array}{l}{ }^{*} \text { "Vehículos } \\
\text { aéreos no } \\
\text { tripulados } \\
\text { "Vehículos } \\
\text { terrestres no } \\
\text { tripulados } \\
\text { "Redes } \\
\text { inalámbricas } \\
\text { virtuales } \\
{ }^{*} \text { Redes } 5 \mathrm{G}\end{array}$ \\
\hline $\begin{array}{l}\text { Algoritmos } \\
\text { metaheurísti } \\
\text { cos }\end{array}$ & $\begin{array}{l}{ }^{*} \text { Algoritmos de } \\
\text { enjambres } \\
{ }^{*} \text { Algoritmos } \\
\text { bioinspirados }\end{array}$ & Media & Media & $\begin{array}{c}\text { Colaborativa } \\
\text { No } \\
\text { colaborativa }\end{array}$ & $\begin{array}{l}\text { No } \\
\text { Supervisado }\end{array}$ & $\begin{array}{l}\text { (Abbas et al., } \\
\text { 2015;Bayrakd } \\
\text { ar y Çalhan, } \\
\text { 2018; Bozorg- } \\
\text { Haddad, 2018; } \\
\text { Javaid et al., } \\
\text { 2018; Kaur et } \\
\text { al., 2018; Modi } \\
\text { y Murmu, } \\
\text { 2017; Peška et } \\
\text { al., 2019) }\end{array}$ & $\begin{array}{l}{ }^{*} \text { Transporte } \\
\text { colaborativo } \\
{ }^{*} \text { Optimizació } \\
\text { n de } \\
\text { objetivos } \\
\text { múltiples } \\
\text { *Intercambio } \\
\text { de } \\
\text { información } \\
{ }^{*} \text { Análisis de } \\
\text { fallas }\end{array}$ \\
\hline $\begin{array}{l}\text { Estrategias } \\
\text { probabilística }\end{array}$ & $\begin{array}{l}{ }^{*} \text { Cadenas de } \\
\text { Markov } \\
{ }^{*} \text { Redes } \\
\text { Bayesianas }\end{array}$ & Alta & Alta & $\begin{array}{c}\text { Colaborativa } \\
\text { No } \\
\text { colaborativa }\end{array}$ & $\begin{array}{l}\text { No } \\
\text { Supervisado }\end{array}$ & $\begin{array}{l}\text { (Rizk et al., } \\
2018 ; \text { Roy et } \\
\text { al., 2017) }\end{array}$ & $\begin{array}{l}{ }^{*} \text { Redes } \\
\text { centralizada } \\
{ }^{*} \text { Robótica } \\
{ }^{*} \text { Redes de } \\
\text { transporte } \\
\text { inteligente }\end{array}$ \\
\hline $\begin{array}{l}\text { Teoría de } \\
\text { juegos }\end{array}$ & $\begin{array}{l}\text { *Juegos } \\
\text { Bayesianos } \\
\text { *Juegos de } \\
\text { Markov } \\
\text { *Juego de } \\
\text { Stackelberg }\end{array}$ & Alta & Alta & $\begin{array}{c}\text { Colaborativa } \\
\text { No } \\
\text { colaborativa }\end{array}$ & $\begin{array}{l}\text { No } \\
\text { Supervisado }\end{array}$ & $\begin{array}{l}\text { (Darwish et al., } \\
\text { 2018; Iftikhar } \\
\text { et al., 2019; } \\
\text { Luce y Raiffa, } \\
\text { 1958; Ren y } \\
\text { Wong, 2019; } \\
\text { Rizk et al., } \\
\text { 2018; Romero } \\
\text { et al., 2019; } \\
\text { Song et al., } \\
\text { 2019; Zaheer } \\
\text { et al., 2018) }\end{array}$ & $\begin{array}{l}{ }^{*} \text { Interferenci } \\
\text { a en las } \\
\text { redes } \\
{ }^{*} \text { Intercambio } \\
\text { de espectro } \\
{ }^{*} \text { Redes } \\
\text { eléctricas } \\
\text { inteligentes } \\
{ }^{*} \text { Computació } \\
\text { n grid }\end{array}$ \\
\hline
\end{tabular}


Tabla 3: (continuación).

\begin{tabular}{|c|c|c|c|c|c|c|c|}
\hline \multirow{2}{*}{ Técnica } & \multirow{2}{*}{ Algoritmo } & \multicolumn{4}{|c|}{ Característica } & \multirow{2}{*}{ Referencia } & \multirow{2}{*}{ Aplicación } \\
\hline & & $C C$ & Precisión & Estrategia & Aprendizaje & & \\
\hline $\begin{array}{l}\text { Algoritmos } \\
\text { de } \\
\text { consensos }\end{array}$ & $\begin{array}{l}\text { *Toma de } \\
\text { decisión } \\
\text { individual } \\
\text { *Toma de } \\
\text { decisión en } \\
\text { grupo }\end{array}$ & Alta & Alta & $\begin{array}{c}\text { Colaborativa } \\
\text { No } \\
\text { colaborativa }\end{array}$ & $\begin{array}{l}\text { Supervisado } \\
\text { No } \\
\text { supervisado }\end{array}$ & $\begin{array}{l}\text { (Luce y Raiffa, } \\
\text { 1958; Wang y } \\
\text { Djurić, 2013; } \\
\text { Zaker et al., } \\
\text { 2018; Zhang } \\
\text { et al. 2019) }\end{array}$ & $\begin{array}{l}\text { *Optimizació } \\
\text { n distribuida } \\
{ }^{*} \text { Optimizació } \\
\text { n de } \\
\text { objetivos } \\
\text { múltiples }\end{array}$ \\
\hline $\begin{array}{l}\text { Toma de } \\
\text { decisiones } \\
\text { multicriterio } \\
\text { (MCDM) }\end{array}$ & $\begin{array}{l}\text { *FFAHP } \\
\text { *FAHP } \\
\text { *AHP } \\
\text { * } \mathrm{SAW} \\
\text { *MEW } \\
{ }^{*} \text { ELECTRE }\end{array}$ & Media & Media & $\begin{array}{c}\text { Colaborativa } \\
\text { No } \\
\text { colaborativa }\end{array}$ & $\begin{array}{l}\text { No } \\
\text { supervisado }\end{array}$ & $\begin{array}{l}\text { (Pedraza et } \\
\text { al., 2019; } \\
\text { Hernández et } \\
\text { al., 2017) }\end{array}$ & $\begin{array}{l}\text { *Movilidad } \\
\text { espectral }\end{array}$ \\
\hline $\begin{array}{l}\text { Series de } \\
\text { tiempo }\end{array}$ & $\begin{array}{l}\text { *AR } \\
\text { *MA } \\
\text { *ARMA } \\
\text { *ARIMA } \\
\text { *ARIMA }\end{array}$ & Alta & Alta & $\begin{array}{c}\text { Colaborativa } \\
\text { No } \\
\text { colaborativa }\end{array}$ & $\begin{array}{l}\text { No } \\
\text { supervisado }\end{array}$ & $\begin{array}{l}\text { (Hernández et } \\
\text { al., 2017) }\end{array}$ & $\begin{array}{l}{ }^{*} \text { Movilidad } \\
\text { espectral } \\
{ }^{*} \text { Control de } \\
\text { acceso al } \\
\text { medio } \\
{ }^{\star} \text { Predicción } \\
\text { oportunidad } \\
\text { espectral }\end{array}$ \\
\hline
\end{tabular}

\section{RETOS EN LA DECISIÓN DE ESPECTRO}

Son muchos los retos presentes en el proceso de toma de decisiones, entre los que se destacan, el tipo de arquitectura (centralizada y descentralizadas), estructuras multiusuario, la externalidad negativa de la red, la implementación en hardware, la complejidad computacional, entre otras, a continuación, se presenta una descripción de algunos de estos retos.

Arquitecturas: en redes de telecomunicaciones, las teorías de toma de decisión permiten solucionar problemas de asignación, sin embargo, como muchas áreas de la ingeniería se ve limitada por el sistema de aplicación. En el caso de las CRN, los modelos desarrollados orientan sus esfuerzos en solucionar problemas de arquitecturas centralizadas (Iftikhar et al., 2019; Tripathi et al., 2019), por lo tanto, es necesario identificar modelos que mejoren el proceso de toma de decisiones para otro tipo de arquitecturas con infraestructura como las arquitecturas descentralizadas.

Multiusuario y externalidad negativa de la red: en las CRN, los SU deben tomar decisiones inteligentes en función de la variación del espectro y de las acciones adoptadas por otros SU, bajo esta dinámica, la probabilidad de que dos o más SU elijan el mismo canal es alta, especialmente cuando el número de SU es mayor que el número de canales disponibles, debido a la externalidad negativa de la red, cuantos más SU seleccionen el mismo canal, menor será la utilidad que cada SU pueda obtener y el número de interferencias por el acceso simultaneo será mayor (Abbas et al., 2015). Para modelar la red bajo parámetros de tráfico realistas, es necesario analizar el acceso de múltiples usuarios de forma simultánea.

Heterogéneidad: para que el proceso de toma de decisiones pueda ser validado correctamente, es necesario realizar la caracterización del espectro bajo parámetros realistas de las condiciones actuales del entorno, por lo tanto, los SU deben observar la disponibilidad de espectro heterogéneo, donde, la heterogeneidad hace referencia a la disponibilidad de espacios espectrales que fluctúan con el tiempo.

Seguridad: con las nuevas estrategias para optimizar el acceso a la red, surgen nuevas aplicaciones y servicios para mejorar la seguridad y privacidad de la información, por lo tanto, se requieren desarrollar nuevas técnicas de criptografía que de acuerdo al nivel de seguridad y privacidad requerido deberán utilizar algoritmos exigentes y eficientes (Romero et al., 2019).

Complejidad computacional y hardware: La optimización del proceso de toma de decisiones es considerado una función no lineal en un espacio de gran dimensión. Por lo tanto, la complejidad computacional es una carga para un hardware, el reto es proponer estrategias que reduzcan esta carga, de tal manera que las propuestas generadas puedan ser probadas en escenarios reales con usuarios reales. 
Finalmente, los desafíos crecen exponencialmente al igual que las técnicas disponibles, es imposible enumerar todos los aprendizajes que pueden surgir dentro de una CRN, por lo tanto, el trabajo permanente en el proceso de toma de decisiones es identificar y proponer algoritmos eficientes (nuevos o híbridos) que permitan abordar tareas más complejas (Rizk et al., 2018; Shi et al., 2018). Además, es necesario desarrollar métricas de evaluación que permitan la comparación de los algoritmos y faciliten su verificación y validación.

\section{CONCLUSIONES}

Este artículo presenta una revisión actual sobre las técnicas de toma de decisiones para CRN. Se analizaron avances recientes en estrategias basadas en inteligencia artificial, específicamente la implementación de máquinas de aprendizaje automático, algoritmos bioinspirados, teoría de juegos y algoritmos de consensos. Los algoritmos presentados en este artículo son una muestra de las herramientas disponibles, en la literatura se encuentran un número amplio de técnicas de toma decisiones aplicadas a otras áreas de la ingeniería que también se pueden aplicar a las CRN.

Para el caso de las CRN distribuidas, los algoritmos bioinspirados, de consenso y la teoría de juegos, presentan una alternativa efectiva para la resolución de las problemáticas presentes en la decisión de espectro debida a la falta de información completa y la complejidad inherente del comportamiento de los modelos descentralizados. Por otro lado, la toma de decisiones multicriterio presenta una alternativa eficiente para CRN centralizadas donde se requieren tiempos de procesamiento bajos y respuestas muy rápidas; en el caso de que se requieran análisis más complejos con altos volúmenes de información, el aprendizaje automático presenta una excelente opción, que últimamente se encuentra en evolución constante.

Aunque los avances son prometedores, aún se deben responder a muchas preguntas. Los algoritmos de toma de decisiones deben aprovechar los avances en inteligencia artificial para obtener mejores resultados, las estrategias deben ser escalables para facilitar la carga computacional y poder resolver problemas de mayor complejidad.

\section{AGRADECIMIENTOS}

Los investigadores resaltan el soporte y financiamiento del proyecto de investigación por parte del Centro de Investigaciones y Desarrollo Científico de la Universidad Distrital Francisco José de Caldas.

\section{REFERENCIAS}

Abbas, N., Nasser, Y., y Ahmad, K. El, Recent advances on artificial intelligence and learning techniques in cognitive radio networks, doi: https://doi.org/10.1186/s13638-015-0381-7, Eurasip Journal on Wireless Communications and Networking 2015(1) (2015)

Alqerm, I., y Shihada, B, Adaptive Decision-Making Scheme for Cognitive Radio Networks. In 2014 IEEE 28th International Conference on Advanced Information Networking and Applications, doi: https://doi.org/10.1109/AINA.2014.41, 321-328 (2014a)

Alqerm, I., y Shihada, B, Adaptive multi-objective Optimization scheme for cognitive radio resource management, doi: https://doi.org/10.1109/GLOCOM.2014.7036916, In 2014 IEEE Global Communications Conference, 857-863 (2014b)

Alqerm, I., y Shihada, B, Hybrid cognitive engine for radio systems adaptation, doi: https://doi.org/10.1109/CCNC.2017.7983233, In 2017 14th IEEE Annual Consumer Communications and Networking Conference (CCNC), 778-783 (2017)

Amjad, M. F., Chatterjee, M., y Zou, C. C, Coexistence in heterogeneous spectrum through distributed correlated equilibrium in cognitive radio networks, doi: https://doi.org/10.1016/j.comnet.2016.01.016, Computer Networks, 98, 109$122(2016)$

Bayrakdar, M. E., y Çalhan, A, Comparative performance evaluation of efficient spectrum handoff methods in wireless cognitive networks, doi: https://doi.org/10.1109/SIU.2018.8404573, In 2018 26th Signal Processing and Communications Applications Conference (SIU), 1-4 (2018)

Bkassiny, M., Li, Y., y Jayaweera, S. K, A Survey on Machine-Learning Techniques in Cognitive Radios, doi: https://doi.org/10.1109/SURV.2012.100412.00017, IEEE Communications Surveys and Tutorials, 15(3), 1136-1159 (2013)

Bernal, C., y Hernández, C, Modelo de decisión espectral para redes de radio cognitiva. UD editorial. Universidad Distrital Francisco José de Caldas, Bogotá, Colombia (2019)

Bozorg-Haddad, O, Advanced optimization by nature-inspired algorithms, doi: https://doi.org/10.1007/978-981-10-5221-7, Springer, 720 (2018) 
CISCO., Cisco Visual Networking Index: Global Mobile Data Traffic Forecast Update. CISCO. Retrieved from https://www.cisco.com/c/en/us/solutions/collateral/service-provider/visual-networking-index-vni/white-paper-c11741490.html (2018).

Darwish, S. M., Saheb, H., y Eltholth, A, A New Game Theory Approach for Noise Reduction in Cognitive Radio Network, doi: https://doi.org/10.1109/ICENCO.2018.8636155, In 2018 14th International Computer Engineering Conference (ICENCO), 84-89 (2018)

Garg, S., Mittal, P., y Nagpal, C. K, Application of game theory to cognitive radio networks for power allocation: An overview, doi: https://doi.org/10.14257/ijhit.2015.8.5.27, International Journal of Hybrid Information Technology, 8(5), 251-258 (2015)

Gochoo, M., Tan, T.-H y otros cinco autores, Novel loT-Based Privacy-Preserving Yoga Posture Recognition System Using Low-Resolution Infrared Sensors and Deep Learning, doi: https://doi.org/10.1109/JIOT.2019.2915095, IEEE Internet of Things Journal, 6(4), 7192-7200 (2019)

Hasegawa, M., Hirai, H., Nagano, K., Harada, H., y Aihara, K, Optimization for centralized and decentralized cognitive radio networks, doi: https://doi.org/10.1109/JPROC.2014.2306255, Proceedings of the IEEE, 102(4), 574-584 (2014)

Hassan, M. H., Hossain, M. J., y Bhargava, V. K, Distributed Beamforming and Autonomous Participation Decision Making in Cooperative CR Systems in Presence of Asynchronous Interference, doi: https://doi.org/10.1109/TWC.2016.2551219, 15(7), IEEE Transactions on Wireless Communications, 5016-5029 (2016)

Hernández, C., Giral, D., y Marquez, H, Evolutive Algorithm for Spectral Handoff Prediction in Cognitive Wireless Networks, doi: https://doi.org/10.12988/ces.2017.7766, HIKARI Ltd, 10(14), 673-689 (2017)

Hernández, C., Pedraza, L., y Martínez, F, Algoritmos para asignación de espectro en redes de radio cognitiva, doi: http://dx.doi.org/10.14483/udistrital.jour.tecnura.2016.2.a05, Tecnura, 20(48), 69-88 (2016).

Hernandez, C., Marquez, H., y Giral, D, Comparative Evaluation of Prediction Models for Forecasting Spectral Opportunities, doi: https://doi.org/10.21817/ijet/2017/v9i5/170905055, International Journal of Engineering and Technology (IJET), 9(5), 3775-3782 (2017)

Hernández, C., Páez, I., y Giral, D, Modelo adaptativo multivariable de handoff espectral para incrementar el desempeño en redes móviles de radio cognitiva, Editorial UD, Primera Edición. Colombia (2017)

Iftikhar, A., Rauf, Z., Ahmed Khan, F., Shoaib Ali, M., y Kakar, M, Bayesian game-based user behavior analysis for spectrum mobility in cognitive radios, doi: https://doi.org/10.1016/j.phycom.2018.12.002, Physical Communication, 32, 200-208 (2019)

Javaid, N., Sher, A., Nasir, H., y Guizani, N, Intelligence in loT-Based 5G Networks: Opportunities and Challenges, doi: https://doi.org/10.1109/MCOM.2018.1800036, IEEE Communications Magazine, 56(10), 94-100 (2018)

Jayaweera, S. K, Signal processing for cognitive radios. John Wiley y Sons (2014)

Jiang, C., Chen, Y., y Liu, K. J. R, Sequential multi-channel access game in distributed cognitive radio networks, doi: https://doi.org/10.1109/GlobalSIP.2014.7032322, In 2014 IEEE Global Conference on Signal and Information Processing (GlobalSIP),1247-1251 (2014b)

Kaur, A., Kaur, A., y Sharma, S, PSO based Multiobjective Optimization for parameter adaptation in CR based loTs, doi: https://doi.org/10.1109/CIACT.2018.8480298, In 2018 4th International Conference on Computational Intelligence and Communication Technology (CICT), 1-7 (2018)

$\mathrm{Li}, \mathrm{Y}$., Shen, H., y Wang, M, Optimization spectrum decision parameters in CR using autonomously search algorithm, doi: https://doi.org/10.1109/ICSP.2016.7878007, In International Conference on Signal Processing (ICSP), 1146-1151 (2016)

López, D., Rivas, E., y Gualdron, O, Primary user characterization for cognitive radio wireless networks using a neural system based on Deep Learning, doi: https://doi.org/10.1007/s10462-017-9600-4, Artificial Intelligence Review (2017)

López Sarmiento, D. L, Implementación de un modelo predictor para la toma de decisiones en redes inalámbricas de radio cognitiva. Universidad Distrital Francico José de Caldas. Retrieved from http://doctoradoingenieria.udistrital.edu.co/index.php/es/investigacion/publicaciones (2017)

Luce, R. D., y Raiffa, H, Games and decisions: Introduction and critical survey. (Dover Publications, Ed.). Wiley New York (1958)

Marquez, H., Hernández, C., y Giral, D, Channel Availability Prediction in Cognitive Radio Networks Using Naive Bayes, doi: https://doi.org/10.12988/ces.2017.7758, HIKARI Ltd, 10(12), 593-605 (2017)

Masonta, M. T., Mzyece, M., y Ntlatlapa, N, Spectrum Decision in Cognitive Radio Networks: A Survey, doi: https://doi.org/10.1109/SURV.2012.111412.00160, IEEE Communications Surveys and Tutorials, 15(3), 1088-1107 (2013)

Mitola, J., y Maguire Jr., G. Q, Cognitive radio: making software radios more personal, doi: https://doi.org/10.1109/98.788210, Personal Communications IEEE, 6(4), 13-18 (1999)

Modi, S., y Murmu, M. K, Multi-SUs interference avoidance spectrum handover algorithm using ant colony method in cognitive radio networks, doi: https://doi.org/10.1109/ICCCNT.2017.82040521-7, In 2017 8th International Conference on Computing, Communication and Networking Technologies (ICCCNT) (2017) 
Morgenstern, O., y Von Neumann, J, Theory of games and economic behavior. Princeton university press (1953)

Murmu, M. K., y Singh, A. K, A bio-inspired leader election protocol for cognitive radio networks, doi: https://doi.org/10.1007/s10586-017-1677-7, Cluster Computing, 22(1), 1665-1678 (2019)

Nguyen, C. Van, y Ahn, H, Distributed Solving Exact Potential Games via Differential Inclusions and Consensus Algorithms, doi: https://doi.org/10.1109/CDC.2018.8619491, In 2018 IEEE Conference on Decision and Control (CDC), 4212-4217 (2018)

Pedraza, L., Hernández, C., y Salgado, C, Modelo de predicción de la ocupación espectral para el análisis y diseño de redes de radio cognitiva. UD editorial. Universidad Distrital Francisco José de Caldas, Bogotá, Colombia (2019)

Peška, L., Tashu, T. M., y Horváth, T, Swarm intelligence techniques in recommender systems - A review of recent research, doi: https://doi.org/https://doi.org/10.1016/j.swevo.2019.04.003, Swarm and Evolutionary Computation, 48, 201$219(2019)$

Pinto, L. R. M., y Correia, L. H. A, Analysis of Machine Learning Algorithms for Spectrum Decision in Cognitive Radios, doi: https://doi.org/10.1109/ISWCS.2018.8491060, In 2018 15th International Symposium on Wireless Communication Systems (ISWCS), 1-6 (2018)

Ramzan, M. R., Nawaz, N., Ahmed, A., Naeem, M., Iqbal, M., y Anpalagan, A, Multi-objective optimization for spectrum sharing in cognitive radio networks: A review, doi: https://doi.org/https://doi.org/10.1016/j.pmcj.2017.07.010, 41(Supplement C), Pervasive and Mobile Computing, 106-131 (2017)

Ren, J., y Wong, K, Cognitive Radio Made Practical: Forward-Lookingness and Calculated Competition, doi: https://doi.org/10.1109/ACCESS.2018.2886607, IEEE Access, 7, 2544-2563 (2019)

Rizk, Y., Awad, M., y Tunstel, E. W, Decision Making in Multi-Agent Systems: A Survey, doi: https://doi.org/10.1109/TCDS.2018.2840971, IEEE Transactions on Cognitive and Developmental Systems, 10(3), 1-15 (2018)

Romero, E., Blesa, J., y Araujo, A, An adaptive energy aware strategy based on game theory to add privacy in the physical layer for cognitive WSNs, doi: https://doi.org/10.1016/j.adhoc.2018.10.026, Ad Hoc Networks, 92, Artículo 101800 (2019)

Roy, A., Midya, S., Majumder, K., Phadikar, S., y Dasgupta, A, Optimized secondary user selection for quality of service enhancement of Two-Tier multi-user Cognitive Radio Network: A game theoretic approach, doi: https://doi.org/10.1016/j.comnet.2017.05.002, Computer Networks, 123, 1-18 (2017)

Safwat, M. A., y Elbadawy, H, Strategic behaviour for spectrum access in hybrid overlay/underlay cognitive radio networks, doi: https://doi.org/10.1049/iet-com.2017.0898, IET Communications, 12(9), 1108-1115 (2018)

Shattal, M. A., Wisniewska, A., Al-Fuqaha, A., Khan, B., y Dombrowski, K, Evolutionary Game Theory Perspective on Dynamic Spectrum Access Etiquette, doi: https://doi.org/10.1109/ACCESS.2017.2736520, IEEE Access, 6, 13142-13157 (2018)

Shi, Y., Sagduyu, Y. E., Erpek, T., Davaslioglu, K., Lu, Z., y Li, J. H, Adversarial Deep Learning for Cognitive Radio Security: Jamming Attack and Defense Strategies, doi: https://doi.org/10.1109/ICCW.2018.8403655, In 2018 IEEE International Conference on Communications Workshops (ICC Workshops), 1-6 (2018)

Song, J. Bin, Li, H., y Coupechoux, M, Game Theory for Networking Applications. Springer (2019)

Sutton, R. S., y Barto, A. G, Reinforcement learning: An introduction. MIT press, EEUU (2018).

Tabassam, A. A., y Suleman, M. U, Game theory in wireless and cognitive radio networks - Coexistence perspective, doi: https://doi.org/10.1109/ISWTA.2012.6373837, In 2012 IEEE Symposium on Wireless Technology and Applications (ISWTA), 177-181 (2012)

Tripathi, S., Upadhyay, A., Kotyan, S., y Yadav, S, Analysis and Comparison of Different Fuzzy Inference Systems Used in Decision Making for Secondary Users in Cognitive Radio Network, doi: https://doi.org/10.1007/s11277-018-6075-9, Wireless Personal Communications, 104(3), 1175-1208 (2019)

Tuberquia-David, L. M., López, H., y Hernández, C, A Multifractal Model for Cognitive Radio Networks. UD editorial. Universidad Distrital Francisco José de Caldas, Bogotá, Colombia (2019)

Wang, C., Chen, Y., y Liu, K. J. R, Hidden Chinese Restaurant Game: Grand Information Extraction for Stochastic Network Learning, doi: https://doi.org/10.1109/TSIPN.2017.2682799, IEEE Transactions on Signal and Information Processing over Networks, 3(2), 330-345 (2017)

Wang, Y, y Djurić, P. M, Reaching Bayesian consensus in cognitive systems by decision exchanges. In 21st European Signal Processing Conference (EUSIPCO 2013), ISBN: 978-0-9928626-0-2, 1-5 (2013)

Wang, Yonghua, Ye, Z., Wan, P., y Zhao, J, A survey of dynamic spectrum allocation based on reinforcement learning algorithms in cognitive radio networks, doi: https://doi.org/10.1007/s10462-018-9639-x, Artificial Intelligence Review, 51(3), 493-506 (2019)

Wu, G., Mallipeddi, R., y Suganthan, P. N, Ensemble strategies for population-based optimization algorithms - A survey, doi: https://doi.org/https://doi.org/10.1016/j.swevo.2018.08.015, Swarm and Evolutionary Computation, 44, 695-711

(2019) 
Yau, K. A., Komisarczuk, P., y Teal, P. D, Applications of Reinforcement Learning to Cognitive Radio Networks, doi: https://doi.org/10.1109/ICCW.2010.5503970, In 2010 IEEE International Conference on Communications Workshops, 1$6(2010)$

Zaheer, K., Othman, M., Rehmani, M. H., y Perumal, T, A Survey of Decision-Theoretic Models for Cognitive Internet of Things (CloT), doi: https://doi.org/10.1109/ACCESS.2018.2825282, IEEE Access, 6, 22489-22512 (2018)

Zaker, N. A., Alsaleem, N., y Kashmoola, M. A, Multi-agent Models Solution to Achieve EMC in Wireless Telecommunication Systems, doi: https://doi.org/10.1109/AiCIS.2018.00061, In 2018 1st Annual International Conference on Information and Sciences (AiCIS), 311-314 (2018)

Zhang, B., Chen, Y., Wang, C.-Y., y Ray Liu, K. J. A, Chinese restaurant game for learning and decision making in cognitive radio networks, doi: https://doi.org/https://doi.org/10.1016/j.comnet.2015.08.010, Computer Networks, 91, 117-134 (2015)

Zhang, H., Dong, Y., Chiclana, F., y Yu, S, Consensus efficiency in group decision making: A comprehensive comparative study and its optimal design, doi: https://doi.org/https://doi.org/10.1016/j.ejor.2018.11.052, European Journal of Operational Research, 275(2), 580-598 (2019) 